\title{
What is new about diet in hepatic encephalopathy
}

\author{
Manuela Merli $^{1} \cdot$ Valerio Iebba $^{2} \cdot$ Michela Giusto $^{1}$
}

Received: 30 July 2015 / Accepted: 15 September 2015

(C) Springer Science+Business Media New York 2015

\begin{abstract}
There is a relationship between hepatic encephalopathy (HE) protein malnutrition and muscle wasting. Muscle may play an alternative role in ammonia detoxification. Molecular mechanisms responsible for muscle depletion are under investigation. Specific nutrients may interact to reverse the molecular pathways involved in muscle wasting at an early stage. Training exercises have also been proposed to improve skeletal muscle mass. However, these data refer to small groups of patients. The amelioration of muscle mass may potentially help to prevent HE. The pathogenesis of HE is associated with modifications of the gut microbiota and diet is emerging to play a relevant role in the modulation of the gut milieu. Vegetarian and fibre-rich diets have been shown to induce beneficial changes on gut microbiota in healthy people, with reduction of Bacteroides spp., Enterobacteriaceae, and Clostridium cluster XIVa bacteria. By way of contrast, it has been suggested that a high-fat or protein diet may increase Firmicutes and reduce Bacteroidetes phylum. Milk-lysozyme and milk-oligosaccharides have also been proposed to induce a "healthy" microbiota. At present, no studies have been published describing the modification of the gut microbiota in cirrhotic patients with $\mathrm{HE}$ as a response to specific diets. New research is needed to evaluate the potentiality of foods in the modulation of gut microbiota in liver disease and HE.
\end{abstract}

Manuela Merli

manuela.merli@uniroma1.it

1 Gastroenterology, Department of Clinical Medicine, "Sapienza" University of Rome, Rome, Italy

2 Department of Public Health and Infectious Diseases, "Sapienza" University of Rome, Rome, Italy
Keywords Cirrhosis $\cdot$ Sarcopenia $\cdot$ Nutrition $\cdot$ Gut microbiota $\cdot$ Vegetables $\cdot$ Fibres

\section{Malnutrition and HE: what correlation?}

Hepatic encephalopathy (HE), together with malnutrition, are among the most frequent complications of liver cirrhosis and upcoming evidence suggests that they may be strictly connected. Indeed, protein malnutrition represents an independent prognostic factor for survival in patients with liver cirrhosis (Merli et al. 1996; Montano-Loza et al. 2012) and is associated with a higher frequency of complications. In a vast series of cirrhotic patients, episodes of hepatic encephalopathy (HE) were reported only in those with a protein-malnutrition diagnosis (Huisman et al. 2011). Furthermore, in a recent prospective study cirrhotic patients with muscle depletion were those at higher risk of both minimal and overt HE (Merli et al. 2013a).

The rationale for the relationship between muscle and HE is based on the role played by muscle in ammonia metabolism. Due to the inability of the failing liver to affect urea synthesis, the muscle plays a crucial role in ammonia detoxification by glutamine synthesis (Olde Damink et al. 2002). Although this metabolic pathway does not result in a definitive ammonia disposal, it has been proposed that muscle depletion may have relevant implications in favouring HE (Wright et al. 2011). From this point of view, amelioration of nutritional status needs to be considered an effective goal to improve the cognitive impairment in these patients. As a consequence, modulation of diet may be considered a promising option when seeking to prevent HE.

European and American Clinical practice guidelines provide clear statements regarding nutritional care in cirrhotic patients (Vilstrup et al. 2014) underlining the fact that the main 
aim of the nutritional support in the presence of liver cirrhosis is to avoid malnutrition and prevent muscle wasting by maintaining an adequate calorie and protein intake. However, in spite of these indications, muscle wasting is still reported in $40-76 \%$ of these patients (Montano-Loza et al. 2012; Giusto et al. 2015; Tandon et al. 2012). It is likely that the dietary approach needs to be associated with something else.

\section{Diet to prevent muscle wasting: should we add something else?}

Knowledge concerning the molecular mechanisms involved in muscle wasting has increased, mainly following studies on experimental models, while few data have appeared regarding cirrhotic patients. Myostatin has been shown to play a central role as a negative regulator of muscle mass although controversial results have been published on muscle biopsies from cirrhotic patients (Qiu et al. 2013; Merli et al. 2013b). Experimental models of biliary cirrhosis (Shih-Yi Lin et al. 2004) suggest that the ubiquitin-proteasome system, which becomes hyper-activated in catabolic states associated with muscle depletion, may be involved in skeletal muscle depletion. However, Porto-caval shunt and muscle biopsies from cirrhotic patients seem to suggest that the expression of critical proteasome components such as MuRF1 and atrogin were unaltered, while expression of autophagy markers have been shown to increase significantly (Dasarathy et al. 2004; Dasarathy et al. 2007, Qiu et al. 2012). An opportunity to explore the issue is provided by supplementing the patient's diet with something capable of counteracting the molecular mechanisms involved in sarcopenia.

In a recent paper, Dasarathy et al. investigated the effect of a single oral branched chain amino acid mixture enriched with leucine (BCAA/LEU) on a small group of alcoholic patients with cirrhosis. Muscle biopsies were obtained before and $7 \mathrm{~h}$ after BCAA/LEU administration. Although the supplementation did not modify the myostatin expression, autophagy markers decreased while the mammalian target of rapamycin (mTOR), regulating protein synthesis, was activated. These data are interesting because they show the potentially positive effect of BCAA/LEU dietary supplementation on the molecular mechanisms responsible for muscle depletion in liver cirrhosis (Tsien et al. 2015). Nevertheless, the interpretation of the results is limited by the lack of an appropriate control group receiving a comparable nitrogen load.

Another possibility to take into consideration is the association of exercise training and a balanced oral diet. In fact training is known to exert a positive effect on muscle mass even in patients with chronic diseases (Zinna and Yarasheski 2003).

In a recent prospective pilot study by Zenith et al., eight weeks of supervised aerobic exercise training increased muscle mass, expressed as thigh circumference, and reduced fatigue in 8 patients with Child-Pugh class A or B cirrhosis (Zenith et al. 2014). Another recent randomized pilot study including cirrhotic patients, Child-Pugh grade A or $\mathrm{B}$, examined whether an exercise programme combined with leucine supplementation might improve patient outcome over a period of 12 weeks. At the end of the period the authors reported that the intervention group ( 8 patients) had improved their exercise capacity (evaluated through a 6 min walk test and 2 min step test) and muscle mass (expressed as lower thigh circumference) (Román et al. 2014).

These results, although based on a small series, suggest that a combined diet, nutritional-supplementation and exercise approach may lead to an improvement in skeletal muscle mass in cirrhotic patients.

Whether the amelioration of muscle mass can reduce or even prevent episodes of HE has not been evaluated and needs to be explored by future studies.

\section{The influence of diet on microbiota: one possible mode of HE prevention or treatment}

In recent years attention has been focused on gut microbiota and its pathogenic role in the development of systemic inflammation, endotoxemia, and portal vasodilation leading to the main complications involved in liver cirrhosis such as HE (Bajaj et al. 2014). A limited number of studies investigating the gut-liver-brain axis in cirrhosis have proposed that gut microbiome composition may impact on the neurocognitive function (Bajaj 2014; Rai et al. 2015). By sequencing bacterial the 16S rDNA gene from the faeces of cirrhotic patients with and without minimal HE (MHE), Zhang and colleagues observed an abundance of Streptococcaceae and Veillonellaceae in cirrhotic patients vs normal individuals. At the same time, they reported that the gut urease-containing species Streptococcus salivarius was present only in cirrhotic patients and not in control subjects. The abundance of Streptococcus salivarius was significantly higher in cirrhotic subjects with MHE respect to those without MHE and was found to be correlated with ammonia levels (Zhang et al. 2013). By way of contrast, Bajaj and coauthors, analyzing the stool microbiota of cirrhotic patients with or without overt HE failed to find significant differences. However, when the colonic mucosa microbiota was investigated, these authors observed a number of differences between patients with and without overt HE. Specifically, Firmicutes phylum and members of Veillonella, Megasphaera, Bifidobacterium, and Enterococcus genera were higher in the HE group, whereas Roseburia was more abundant in the non-HE group (Bajaj et al. 2012).

The identification of a close connection between the gut microbiota, the liver and the brain, together with the 
awareness that diet may play a crucial role in the regulation of the gut milieu, led to the hypothesis that modulation of the gut microbiota through changes in the quality of food assumption may be a potential therapeutic target for HE.

One of the first studies, aimed at investigating whether a dietary supplement capable of modulating gut microbiota might prove useful in the treatment of MHE and prevention of overt HE, utilized a probiotic yogurt. The cirrhotic patients were randomized to receive $12 \mathrm{oz}$ of yogurt daily in addition to dietary recommendations to follow for 60 days. MHE improved in a higher percentage of the patients randomized to supplementation and in the same group the occurrence of overt HE was prevented as compared to the non-supplemented patients (Bajaj et al. 2008). Many other studies have utilized the addition of prebiotics, probiotics and symbiotics, defined as functional food components, to daily diet for the management of HE (Amodio et al. 2013).

\section{Vegetarian and fibre-rich diet}

Information on the effect of different diets on gut microbiota in liver disease is still not available (Giannelli et al. 2014), however, the idea that diet may represent a reliable alternative therapy in the treatment of HE through modulation of the microbiota is a really fascinating perspective. This working hypothesis provides a new way to explain the positive effects reported for vegetarian diets in patients with cirrhosis and HE (Amodio et al. 2001). In the course of the past few decades it has been claimed that vegetable proteins are better tolerated than animal proteins in cirrhotic patients with HE (Merli et al. 2012). Some of the proposed beneficial effects are the higher intestinal clearance of nitrogen-waste products, due to the high-fibre content which is capable of inducing a greater bacterial mass, a shortened transit time, and a reduced colonic $\mathrm{pH}$ entrapping ammonia in the intestinal lumen, a higher ornithine and arginine content, which may facilitate ammonia disposal through the urea cycle, and a lower methionine and tryptophan content compared to animal proteins. Nowadays new evidence suggests that additional benefits of a vegetalenriched diet may derive from the modulation of gut microbiota. At present, studies evaluating the effects of a vegetable enriched diet on gut microbiota are available only for healthy people. In a study by Zimmer et al., a reduction in Bacteroides spp., Bifidobacterium spp., Escherichia coli and Enterobacteriaceae was observed in fecal samples of patients following a vegetarian/vegan diet vs patients on an omnivorous diet (Zimmer et al. 2011). In a cohort of vegetarian and omnivorous young women in southern India, faecal microbiota was found to be enriched with Clostridium cluster XIVa bacteria, specifically Roseburia and $E$. rectale, in the omnivorous group (Kabeerdoss et al. 2012). By contrast, other authors failed to report any differences in the microbiota composition of subjects following the these diets (Liszt et al. 2009). The variability of the results provided by the studies available may derive from the heterogeneity of the population and the acknowledge that even a vegetable enriched diet may vary in daily composition, inducing a rapid change in the gut microbiome (David et al. 2014). New and well defined studies on the effect of vegetarian/vegetables-enriched diets on the composition of gut microbiota in cirrhotic patients are needed. The impact of diets rich in whole-grain bread, cereals or rice compared to those comprising white wheat grain have also been investigated. A diet enriched by whole grain products, with high fibre content, seems to induce an increase in Bifidobacteria and a reduction of Bacteroidetes in gut microbiota (Costabile et al. 2008; Martínez et al. 2013; Lappi et al. 2013). The microbiota of children following the low-protein, carbohydrate, "Fiber-based diet", typical of rural countries as opposed to the "protein and polysaccharide-rich diet" typical of the western countries, shows a significant enrichment in Bacteroidetes and a depletion in Firmicutes (De Filippo et al. 2010). Enterobacteriaceae (Shigella and Escherichia genera) were also significantly lower in the "Fibre-based diet" as opposed to the "protein and polysaccharide-rich diet".

These dietary approaches might provide an interesting opportunity to modulate gut microbiota in cirrhotic patients.

\section{High fat diet}

If a beneficial effect on the gut microbiota has been suggested after the introduction of vegetables or fibres, high-fat diet has been shown to induce a shift in the balance between the two major phyla of gut microbiota, leading to an increase of Firmicutes and a decrease of Bacteroidetes (Hildebrandt et al. 2009; Turnbaugh et al. 2009). The administration of cholic acid to rats induced phylum-level alteration in the composition of the gut microbiota with an increase in Firmicutes and a reduction in Bacteroidetes, which are very similar to the changes induced by a high-fat diet (Islam et al. 2011). Given that bile acid secretion increases following a high-fat diet (Reddy 1981), the authors speculated that bile acids might be a determinant of the gut microbiota in response to a highfat diet (Islam et al. 2011; Yokota et al. 2012). The possible modifications induced by a high-fat diet in cirrhotic patients needs, therefore, also to take the alteration in biliary secretion in these patients into account too.

\section{Specific nutrients improving "healthy microbiota"}

Some interesting studies have focused their attention on the influence of specific food constituents on the promotion of "a healthy-microbiota" (Maga et al. 2013; Barile and Rastall 2013). Among these food constituents, milk-lysozyme and milk-oligosaccharides have been investigated. Lysozyme is a 
Fig. 1 How can diet contribute to ameliorate hepatic encephalopathy

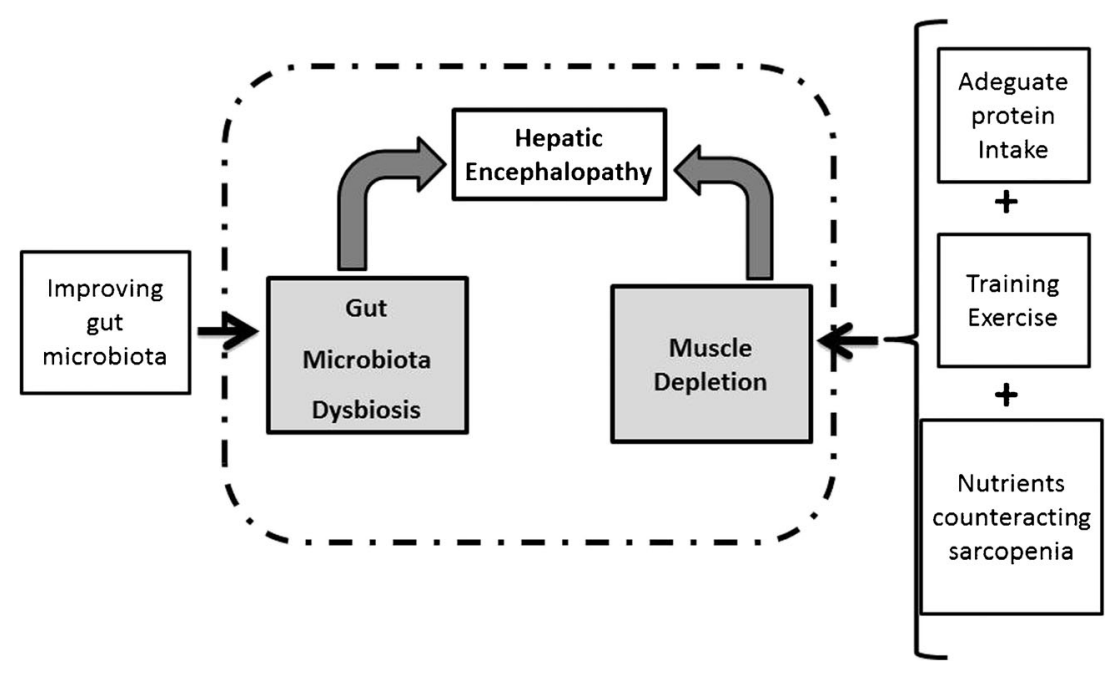

naturally occurring antimicrobial enzyme found in the tears, saliva, and milk of all mammals that lyses a specific link in the peptidoglycan layer of bacterial cell walls. In an experimental study, milk enriched with $68 \%$ human lysozyme was used to feed young pigs. This treatment induced an increase in the bacteria associated with gut health (Bifidobacteriaceae and Lactobacillaceae) and a decrease in those associated with diseases (Mycobacteriaceae, Streptococcaceae, Campylobacterales) vs the control-fed group (Maga et al. 2012). This study demonstrated that a single component of the diet, with bioactivity, may change the microbiome composition of the gut. Other studies have investigated the role of Human Milk Oligosaccharides (HMO): HMO are being recognized as a new class of potent bioactive molecules capable of enhancing the growth of Bifidobacteria and Lactobacilli, reducing harmful bacteria such as Clostridia, Enterococci, Eubacteria and Enterobacteria, and preventing bacteria from attaching to target oligosaccharides on the intestinal mucosal surface (Fucosylated and sialylated oligosaccharides) (German et al. 2008; Bode 2012). Recent research has demonstrated that bovine milk contains oligosaccharides that are analogous to HMO and perform a similarly protective role (Zivkovic and Barile 2011). However, fluid bovine milk contains only trace amounts of oligosaccharides; for that reason, in order to increase the concentration of such oligosaccharides, a whey permeate has been produced from bovine milk and has been the subject of recent investigations. Whey permeate, further processed by membrane filtration, may have as much as 10 -fold higher total concentrations of free oligosaccharide than bovine milk (Zivkovic and Barile 2011) and may represent a promising alternative as functional food to induce a healthy gut microbiota.

All the data reported suggest that by increasing our understanding we will be able in the future to modulate the gut microbiota through the introduction of specific foods or by adopting specific diets. Fresh research in this field concerning liver patients is required in order to understand how to take advantage of the great potentiality of foods in the modulation of the gut microbiota, in order to provide a therapeutic strategy capable of treating or even of preventing complications such as hepatic encephalopathy in those suffering from cirrhosis.

In conclusion, diet may play a central role as therapeutic support in the treatment and prevention of HE not only by counteracting malnutrition, but also by exerting an effect on others pathogenic mechanisms responsible for HE. Among these, new targets are represented by reversal of sarcopenia and by the modulation of the gut microbiota (Fig. 1).

Conflict of interest The authors declare that they have no conflict of interest.

\section{References}

Amodio P, Caregaro L, Pattenò E, Marcon M, Del Piccolo F, Gatta A (2001) Vegetarian diets in hepatic encephalopathy: facts or fantasies? Dig Li ver Dis 33(6):492-500

Amodio P, Bemeur C, Butterworth R, Cordoba J, Kato A, Montagnese S, Uribe M, Vilstrup H, Morgan MY (2013) The nutritional management of hepatic encephalopathy in patients with cirrhosis: international society for hepatic encephalopathy and nitrogen metabolism consensus. Hepatology 58(1):325-336

Bajaj JS (2014) The role of microbiota in hepatic encephalopathy. Gut Microbes 5(3):397-403

Bajaj JS, Saeian K, Christensen KM, Hafeezullah M, Varma RR, Franco J, Pleuss JA, Krakower G, Hoffmann RG, Binion DG (2008) Probiotic yogurt for the treatment of minimal hepatic encephalopathy. Am J Gastroenterol 103:1707-1715

Bajaj JS, Hylemon PB, Ridlon JM, Heuman DM, Daita K, White MB, Monteith P, Noble NA, Sikaroodi M, Gillevet PM (2012) Colonic mucosal microbiome differs from stool microbiome in cirrhosis and hepatic encephalopathy and is linked to cognition and inflammation. Am J Physiol Gastrointest Liver Physiol 303(6):G675-GG85

Bajaj JS, Heuman DM, Hylemon PB, Sanyal AJ, White MB, Monteith P, Noble NA, Unser AB, Daita K, Fisher AR, Sikaroodi M, Gillevet 
PM (2014) Altered profile of human gut microbiome is associated with cirrhosis and its complications. J Hepatol 60(5):940-947

Barile D, Rastall RA (2013) Human milk and related oligosaccharides as prebiotics. Curr Opin Biotechnol 24:214-219

Bode L (2012) Human milk oligosaccharides: every baby needs a sugar mama. Glycobiology 22(9):1147-1162

Costabile A, Klinder A, Fava F, Napolitano A, Fogliano V, Leonard C, Gibson GR, Tuohy KM (2008) Whole-grain wheat breakfast cereal has a prebiotic effect on the human gut microbiota: a double-blind, placebo-controlled, crossover study. Br J Nutr 99(1):110-120

Dasarathy S, Dodig M, Muc SM, Kalhan SC, McCullough AJ (2004). Skeletal muscle atrophy is associated with an increased expression of myostatin and impaired satellite cell function in the portacaval anastamosis rat. Am J Physiol Gastrointest Liver Physiol 287: G1124-G1130

Dasarathy S, Muc S, Hisamuddin K, Edmison JM, Dodig M, McCullough AJ, Kalhan SC (2007). Altered expression of genes regulating skeletal muscle mass in the portacaval anastomosis rat. Am J Physiol Gastrointest Liver Physiol 292: G1105-G1113.

David LA, Maurice CF, Carmody RN, Gootenberg DB, Button JE, Wolfe BE, Ling AV, Devlin AS, Varma Y, Fischbach MA, Biddinger SB, Dutton RJ, Turnbaugh PJ (2014) Diet rapidly and reproducibly alters the human gut microbiome. Nature 505(7484):559-563

De Filippo C, Cavalieri D, Di Paola M, Ramazzotti M, Poullet JB, Massart S, Collini S, Pieraccini G, Lionetti P (2010) Impact of diet in shaping gut microbiota revealed by a comparative study in children from Europe and rural Africa. Proc Natl Acad Sci U S A 107: 14691-14696

German JB, Freeman SL, Lebrilla CB, Mills DA (2008) Human milk oligosaccharides: evolution, structures and bioselectivity as substrates for intestinal bacteria. Nestle Nutr Workshop Ser Pediatr Program 62:205-218

Giannelli V, Di Gregorio V, Iebba V, Giusto M, Schippa S, Merli M, Thalheimer U (2014) Microbiota and the gut-liver axis: bacterial translocation, inflammation and infection in cirrhosis. World $\mathrm{J}$ Gastroenterol 20(45):16795-16810

Giusto M, Lattanzi B, Albanese C, Galtieri A, Farcomeni A, Giannelli V, Lucidi C, Di Martino M, Catalano C, Merli M (2015) Sarcopenia in liver cirrhosis: the role of computed tomography scan for the assessment of muscle mass compared with dual-energy X-ray absorptiometry and anthropometry. Eur J Gastroenterol Hepatol 27:328-334

Hildebrandt MA, Hoffmann C, Sherrill-Mix SA, Keilbaugh SA, Hamady M, Chen YY, Knight R, Ahima RS, Bushman F, Wu GD (2009) High-fat diet determines the composition of the murine gut microbiome independently of obesity. Gastroenterology 137(5): 1716-1724

Huisman EJ1, Trip EJ, Siersema PD, van Hoek B, van Erpecum KJ (2011) Protein energy malnutrition predicts complications in liver cirrhosis. Eur J Gastroenterol Hepatol 23:982-989

Islam KB, Fukiya S, Hagio M, Fujii N, Ishizuka S, Ooka T, Ogura Y, Hayashi T, Yokota A (2011) Bile acid is a host factor that regulates the composition of the cecal microbiota in rats. Gastroenterology 141(5):1773-1781

Kabeerdoss J, Devi RS, Mary RR, Ramakrishna BS (2012) Faecal microbiota composition in vegetarians: comparison with omnivores in a cohort of young women in southern India. Br J Nutr 108(6):953957

Lappi J, Salojärvi J, Kolehmainen M, Mykkänen H, Poutanen K, de Vos WM, Salonen A (2013) Intake of whole-grain and fiber-rich rye bread versus refined wheat bread does not differentiate intestinal microbiota composition in Finnish adults with metabolic syndrome. J Nutr 143(5):648-655

Lin SY, Chen WY, Lee FY, Huang CJ, Sheu WH (2004) Activation of ubiquitin-proteasome pathway is involved in skeletal muscle wasting in a rat model with biliary cirrhosis: potential role of TNFalpha. Am J Physiol Endocrinol Metab 288:E493-E501
Liszt K, Zwielehner J, Handschur M, Hippe B, Thaler R, Haslberger AG (2009) Characterization of bacteria, clostridia and Bacteroides in faeces of vegetarians using qPCR and PCR-DGGE fingerprinting. Ann Nutr Metab 54(4):253-257

Maga EA, Weimer BC, Murray JD (2012) Consumption of lysozymerich milk can alter microbial fecal populations. Appl Environ Microbiol 78(17):6153-6160

Maga EA, Bart C. Weimer James D Murray (2013). Dissecting the role of milk components on gut microbiota composition. Gut Microbes 4(2): 136-139.

Martínez I, Lattimer JM, Hubach KL, Case JA, Yang J, Weber CG, Louk JA, Rose DJ, Kyureghian G, Peterson DA, Haub MD, Walter J (2013) Gut microbiome composition is linked to whole graininduced immunological improvements. ISME J 7(2):269-280

Merli M, Riggio O, Dally L (1996) Does malnutrition affect survival in cirrhosis? PINC (policentrica italiana nutrizione cirrosi). Hepatology 23:1041-1046

Merli M, Giusto M, Riggio O (2012). Nutrition and hepatic encephalopathy. In: Mullen KD, Prakash RK (eds) Hepatic Encephalopathy, Springer Science+Business Media, New York, p 99-209

Merli M, Giusto M, Lucidi C, Giannelli V, Pentassuglio I, Di Gregorio V, Lattanzi B, Riggio O (2013a) Muscle depletion increases the risk of overt and minimal hepatic encephalopathy: results of a prospective Study. Metab Brain Dis 28:281-284

Merli M, Giusto M, Molfino A, Bonetto A, Rossi M, Ginanni Corradini S, Baccino FM, Rossi Fanelli F, Costelli P, Muscaritoli M (2013b) MuRF-1 and p-GSK3 $\beta$ expression in muscle atrophy of cirrhosis. Liver Int 33:714-721

Montano-Loza AJ, Meza-Junco J, Prado CM, Lieffers JR, Baracos VE, Bain VG, Sawyer MB (2012) Muscle wasting is associated with mortality in patients with cirrhosis. Clin Gastroenterol Hepatol 10: 166-173

Olde Damink SW1, Deutz NE, Dejong CH, Soeters PB, Jalan R (2002) Interorgan ammonia metabolism in liver failure. Neurochem Int 41(2-3):177-188

Qiu J, Tsien C, Thapalaya S, Narayanan A, Weihl CC, Ching JK, Eghtesad B, Singh K, Fu X, Dubyak G, McDonald C, Almasan A, Hazen SL, Naga Prasad SV, Dasarathy S (2012) Hyperammonemiamediated autophagy in skeletal muscle contributes to sarcopenia of cirrhosis. Am J Physiol Endocrinol Metab 303:E983-E993

Qiu J, Thapaliya S, Runkana A, Yang Y, Tsien C, Mohan ML, Narayanan A, Eghtesad B, Mozdziak PE, McDonald C, Stark GR, Welle S, Naga Prasad SV, Dasarathy S (2013) Hyperammonemia in cirrhosis induces transcriptional regulation of myostatin by an NF- $\mathrm{kB}$ mediated mechanism. Proc Natl Acad Sci U S A 110:18162-18167

Rai R, Saraswat VA, Dhiman RK (2015) Gut microbiota: its role in hepatic encephalopathy. J Clin Exp Hepatol 5:S29-S36

Reddy BS (1981) Diet and excretion of bile acids. Cancer Res 41(9 Pt 2): 3766-3678

Román E, Torrades MT, Nadal MJ, Cárdenas G, Nieto JC, Vidal S, Bascuñana H, Juárez C, Guarner C, Córdoba J, Soriano G (2014) Randomized pilot study: effects of an exercise programme and leucine supplementation in patients with cirrhosis. Dig Dis Sci 59: 1966-1975

Tandon P, Ney M, Irwin I, Ma MM, Gramlich L, Bain VG, Esfandiari N, Baracos V, Montano-Loza AJ, Myers RP (2012) Severe muscle depletion in patients on the liver transplant wait list: its prevalence and independent prognostic value. Liver Transpl 18:1209-1216

Tsien C, Davuluri G, Singh D, Allawy A, Ten Have GA, Thapaliya S, Schulze JM, Barnes D, McCullough AJ, Engelen MP, Deutz NE, Dasarathy S (2015) Metabolic and molecular responses to leucineenriched branched chain amino acid supplementation in the skeletal muscle of alcoholic Cirrhosis. Hepatology 61(6):2018-2029

Turnbaugh PJ, Ridaura VK, Faith JJ, Rey FE, Knight R, Gordon JI (2009) The effect of diet on the human gut microbiome: a metagenomic analysis in humanized gnotobiotic mice. Sci Transl Med 1(6):6ra14 
Vilstrup H, Amodio P, Bajaj J, Cordoba J, Ferenci P, Mullen KD, Weissenborn K, Wong P (2014) Hepatic encephalopathy in chronic liver disease: 2014 practice guideline by the American association for the study of liver diseases and the European association for the study of the liver. Hepatology 60:715-735

Wright G, Noiret L, Olde Damink SW, Jalan R (2011) Interorgan ammonia metabolism in liver failure: the basis of current and future therapies. Liver Int 31:163-175

Yokota A, Fukiya S, Islam KB, Ooka T, Ogura Y, Hayashi T, Hagio M, Ishizuka $S$ (2012) Is bile acid a determinant of the gut microbiota on a high-fat diet? Gut Microbes 3(5):455-459

Zenith L, Meena N, Ramadi A, Yavari M, Harvey A, Carbonneau M, Ma M, Abraldes JG, Paterson HMJ, Tandon P (2014) Eight weeks of exercise training increases aerobic capacity and muscle mass and reduces fatigue in patients with cirrhosis. Clin Gastroenterol Hepatol 12:1920-1926

Zhang Z, Zhai H, Geng J, Yu R, Ren H, Fan H, Shi P (2013) Large-scale survey of gut microbiota associated with MHE Via 16S rRNA-based pyrosequencing. Am J Gastroenterol 108(10):1601-1611

Zimmer J, Lange B, Frick JS, Sauer H, Zimmermann K, Schwiertz A, Rusch K, Klosterhalfen S, Enck P (2011) A vegan or vegetarian diet substantially alters the human colonic faecal microbiota. Eur J Clin Nutr 66(1):53-60

Zinna EM, Yarasheski KE (2003) Exercise treatment to counteract protein wasting of chronic diseases. Curr Opin Clin Nutr Metab Care 6:8793

Zivkovic AM, Barile D (2011) Bovine milk as a source of functional oligosaccharides for improving human health. Adv Nutr 3:284-289 